\title{
Genome-wide analysis of transcription factors related to anthocyanin biosynthesis in carmine radish (Raphanus sativus L.) fleshy roots
}

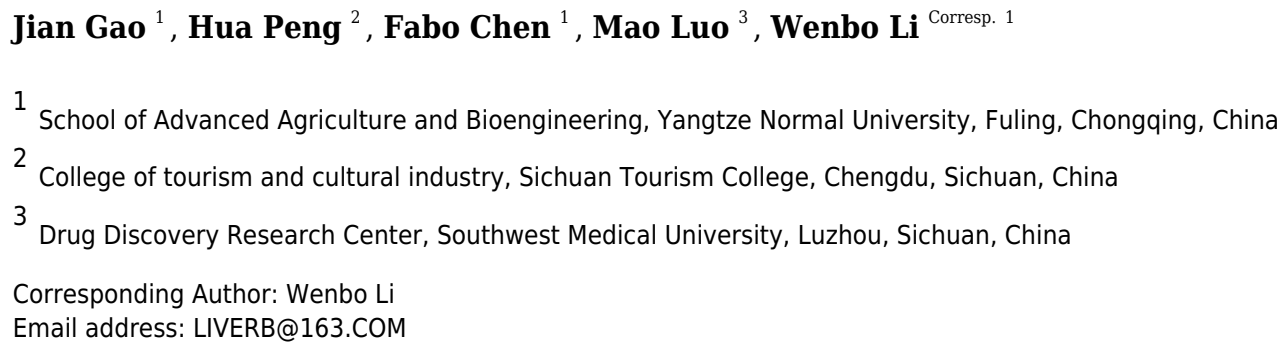

Carmine radish produced in Chongqing is famous for containing a natural red pigment (red radish pigment). However, the anthocyanin biosynthesis transcriptome and the expression of anthocyanin biosynthesis-related genes in carmine radish have not been fully investigated. Uncovering the mechanism of anthocyanin biosynthesis in the 'Hongxin 1' carmine radish cultivar has become a dominant research topic in this field. In this study, a local carmine radish cultivar named 'Hongxin 1' containing a highly natural red pigment was used to analyze transcription factors (TFs) related to anthocyanin biosynthesis during the dynamic development of fleshy roots. Based on RNA sequencing data, a total of 1,747 TFs in 64 TF families were identified according to their DNA-binding domains. Of those, approximately 71 differentially expressed transcription factors (DETFs) were commonly detected in any one stage compared with roots in the seedling stage (SS_root). Moreover, 26 transcripts of DETFs targeted by 74 miRNAs belonging to 25 miRNA families were identified, including MYB, WRKY, bHLH, ERF, GRAS, NF-YA, C2H2-Dof, and HD-ZIP. Finally, 8 DETF transcripts belonging to the C2C2-Dof, bHLH and ERF families and their 8 corresponding miRNAs were selected for qRT-PCR to verify their functions related to anthocyanin biosynthesis during the development of carmine radish fleshy roots. Finally, we propose a putative miRNA-target regulatory model associated with anthocyanin biosynthesis in carmine radish. Our findings suggest that sucrose synthase might act as an important regulator to modulate anthocyanin biosynthesis in carmine radish by inducing several miRNAs (miR165a-5p, miR172b, miR827a, miR166g and miR1432-5p) targeting different ERFs than candidate miRNAs in the traditional WMBW complex in biological processes. 
1 Genome-wide analysis of transcription factors related

2 to anthocyanin biosynthesis in carmine radish

3 (Raphanus sativus L.) fleshy roots

4

5

6

\begin{abstract}
Jian $\mathrm{Gao}^{1 \dagger}$, Hua peng ${ }^{2 \dagger}$, Fabo Chen ${ }^{1}$, Mao Luo ${ }^{3}$ and Wenbo $\mathrm{Li}^{1 *}$
\end{abstract}
${ }^{1}$ School of Advanced Agriculture and Bioengineering, Yangtze Normal University, Fuling, Chongqing, China

${ }^{2}$ College of tourism and cultural industry, Sichuan Tourism College, Chengdu, Sichuan, China

${ }^{3}$ Drug Discovery Research Center, Southwest Medical University, Luzhou, Sichuan, China

$\dagger$ These authors contributed equally to this work.

${ }^{*}$ Correspondence author:

Wenbo $\mathrm{Li}^{1}$

No.16 Juxian Road, Fuling, Chongqing, 408100, China

E-mail adress: corresponding_author_liverb@163.com

\title{
Abstract
}

Carmine radish produced in Chongqing is famous for containing a natural red pigment (red radish pigment). However, the anthocyanin biosynthesis transcriptome and the expression of anthocyanin biosynthesis-related genes in carmine radish have not been fully investigated. Uncovering the mechanism of anthocyanin biosynthesis in the 'Hongxin 1' carmine radish cultivar has become a dominant research topic in this field. In this study, a local carmine radish cultivar named 'Hongxin 1' containing a highly natural red pigment was used to analyze transcription factors (TFs) related to anthocyanin biosynthesis during the dynamic development of fleshy roots. Based on RNA sequencing data, a total of 1,747 TFs in $64 \mathrm{TF}$ families were identified according to their DNA-binding domains. Of those, approximately 71 differentially expressed transcription factors (DETFs) were commonly detected in any one stage compared with roots in the seedling stage (SS_root). Moreover, 26 transcripts of DETFs targeted by 74 miRNAs belonging to 25 miRNA families were identified, including $M Y B, W R K Y, b H L H, E R F$, GRAS, NF-YA, C2H2-Dof, and HD-ZIP. Finally, 8 DETF transcripts belonging to the C2C2-Dof, $b H L H$ and $E R F$ families and their 8 corresponding miRNAs were selected for qRT-PCR to verify their functions related to anthocyanin biosynthesis during the development of carmine radish fleshy roots. Finally, we propose a putative miRNA-target regulatory model associated with anthocyanin biosynthesis in carmine radish. Our findings suggest that sucrose synthase might act as an important regulator to modulate anthocyanin biosynthesis in carmine radish by inducing several miRNAs (miR165a-5p, miR172b, miR827a, miR166g and miR1432-5p) targeting different ERFs than candidate miRNAs in the traditional WMBW complex in biological processes. 
40

41

42

43

44

45

46

47

48

49

50

51

52

53

54

55

56

57

58

59

60

61

62

63

64

65

66

67

68

69

70

71

72

73

74

75

76

77

78

\section{Introduction}

Radish (Raphanus sativus L.), a biennial root vegetable crop of the Brassicaceae family, is an economically important vegetable crop with an edible taproot. Currently, multiple colors have been identified in the flesh of the taproot, including white, yellow, red, black and purple. The carmine radish 'Hongxin', produced in Chongqing, is famous for containing natural red pigment (red radish pigment). Anthocyanins are recognized as regulators of red to purple colors in nature and thereby produce water-soluble pigments belonging to the flavonoid group (Khoo et al. 2017). Most research has demonstrated that anthocyanins, a beneficial food additive worldwide, could pose major public health threats (cardiovascular disease, inflammation, obesity, and diabetes) caused by the chemical synthesis of food additives (He \& Giusti 2010; Yousuf et al. 2015). In addition, most regulatory genes have been extensively found to be involved in the anthocyanin biosynthesis pathway, which is largely conserved among flowering plants(Anju et al. 2017). Structural and regulatory genes could participate in anthocyanin biosynthesis. Previous studies have demonstrated that anthocyanins are first formed from phenylalanine by structural genes that encode a series of enzymes, such as phenylalanine ammonia-lyase $(P A L)$, cinnamic 4hydroxylase $(\mathrm{CHH})$ and 4-coumarate-CoA ligase $(4 \mathrm{CL})$, followed by chalcone synthase $(\mathrm{CHS})$, through phenylpropanoid metabolism. Then, the product $4,2^{\prime} 4^{\prime} 6^{\prime}$-tetrahydrocychalcone is successively catalyzed by four enzymes [Chalcone Isomerase $(C H I)$, flavanone 3-Hydroxylase $(F 3 H)$, dihydroflavonol 4-Reductase $(D F R)$, and anthocyanin synthase $(A N S / L D O X)]$ (AzaGonzález et al. 2012; Dao et al. 2011). Additionally, transcription factors were demonstrated to be important regulatory genes involved in the anthocyanin biosynthetic pathway. Transcription factors (TFs) play vital roles in the normal development of an organism and in routine cellular functions (Latchman 1993; Yusuf et al. 2012). Recently, researchers have demonstrated that MYB-bHLH-WDR (MBW) complexes could transcriptionally regulate genes encoding these enzymes through $M Y B, b H L H$ and $W D 40$ repeats (Xu et al. 2015). In addition, the regulatory genes Jasmonate zim-domain ( $J A Z$ ) (Qi et al. 2011), Squamosa promoter binding protein-like $(S P L)$ (Gou et al. 2011) and $N A C$ (Zhou et al. 2015) have also been reported to regulate anthocyanin biosynthesis. Moreover, recent evidence has shown that microRNAs (miRNAs) can act as important regulators of anthocyanin biosynthesis in plants(Jia et al. 2015b; Yang et al. 2013); examples include the miR156, miR165/166, miR828 and miR858 families in Arabidopsis (Yang et al. 2013) and Solanum lycopersicum(Jia et al. 2015b). However, the associations of these miRNAs with transcription factors (TFs) have not been systematically investigated, and the related miRNAs involved in regulating anthocyanin biosynthesis have not been reported in carmine radish.

In this study, transcriptome analysis and functional validation of putative differentially expressed transcription factors (DETFs) involved in anthocyanin biosynthesis were performed using a local carmine radish cultivar named 'Hongxin 1', which contains a highly natural red pigment. As determined by RNA sequencing, a total of 1,747 TFs comprising 64 TF families were identified as being involved in the dynamic development of fleshy roots according to their 
79 DNA-binding domains. Of these TFs, approximately 71 DETFs were commonly differentially

80 expressed in any one stage compared with SS_root. Of those 71 DETFs, 26 were predicted to be 81 targets of 74 miRNAs belonging to 25 miRNA families, and many DETF gene products cleaved 82 transcripts targeted by miRNAs, including $M Y B, W R K Y, b H L H, E R F, G R A S, N F-Y A, C 2 H 2-D o f$, 83 and $H D$-ZIP. In addition, 8 DETF transcripts belonging to the C2C2-Dof, bHLH and ERF 84 families and their 8 corresponding miRNAs were selected for qRT-PCR to verify their functions 85 in the development of fleshy roots in carmine radish. Finally, a putative miRNA-target regulatory model associated with anthocyanin biosynthesis in carmine radish was illustrated. Our findings suggest that miRNAs are involved in the regulation of anthocyanin biosynthesis in the dynamic development of fleshy roots in carmine radish.

\section{Materials and methods}

\section{Quantification of anthocyanin levels in carmine radish}

Five local cultivars of carmine radish ('Hongxin 1', 'Guanguan', 'Longquan 1', Yanzhi 1' and 'Yanzhi 2') containing natural red pigment (red radish pigment) collected from Fuling were selected as experimental materials. To identify the pigment contents of the five local cultivars of carmine radish, the dynamics of anthocyanin in the development of fleshy roots in carmine radish were investigated by HPLC analysis. The study groups included fleshy roots from the seedling stage (SS, 15 days after planting), initially expanded fleshy roots (IE, 40 days after planting), fully expanded fleshy roots (FE, 70 days after planting), fleshy roots from the bolting stage (BS, 120 days after planting), fleshly roots from the initial flowering stage (IFS, 140 days after planting), fleshy roots from the full-bloom stage (FBS, 160 days after planting), and fleshy roots from the podding stage (PS, 200 days after planting). Briefly, fleshy roots were collected from three homozygous individuals from the five local cultivars of carmine radish and pooled together; three replicates were included. The fleshy root tissues were ground in liquid nitrogen, and the red pigment was then extracted with a solvent mixture containing methanol $(40 \%, \mathrm{v} / \mathrm{v})$, formic acid $(0.1 \%, \mathrm{v} / \mathrm{v})$ and acetone $(40 \%, \mathrm{v} / \mathrm{v})$. We used a $10 \mu \mathrm{L}$ injection volume on a VDS C18 column $(4.6 \times 250 \mathrm{~mm}, 5 \mu \mathrm{m}$, VDS Optilab, Germany) with a $0.8 \mathrm{~mL}$ min- 1 flow rate. Based on the results from HPLC analysis, RNA-Seq was used to investigate young fleshy roots in the carmine radish 'Hongxin 1' cultivar obtained from the dynamic fleshy root developmental stages, including fleshy roots from the seedling stage (SS), the initial expansion (IE) stage, the full expansion (FE) stage, the bolting stage (BS), the initial flowering stage (IFS), the full-bloom stage (FBS) and the podding stage (PS) (Fig. S1). In this study, based on our private RNA sequencing data (unpublished, submitted to Frontiers in Plant Science), transcriptome analysis and functional validation of putative differentially expressed transcription factors (DETFs) related to the development of fleshy roots in 'Hongxin' carmine radish were conducted.

\section{Sample collection and RNA isolation}

The carmine radish 'Hongxin 1' was cultivated in a greenhouse at the experimental farm of Yihe (Yangtze Normal University experiment base) in 2018. First, we sowed seeds of 'Hongxin 1' in sterilized soil for 2 weeks under normal growth conditions $\left(23^{\circ} \mathrm{C}, 16 \mathrm{~h} \mathrm{light} / 8 \mathrm{~h}\right.$ dark $)$. Next, 2 - 
119 week-old plants were transferred and kept for 15 days in the cold room $\left(5 \pm 1{ }^{\circ} \mathrm{C}, 12 \mathrm{~h} \mathrm{light} / 12 \mathrm{~h}\right.$ 120 dark) for vernalization treatment. After the vernalization period, the plants were grown in a 121 normal growth room under normal growth conditions $\left(23^{\circ} \mathrm{C}, 16 \mathrm{~h}\right.$ light $/ 8 \mathrm{~h}$ dark). At least three 122 independent biological replicates of fleshy roots obtained from the development stages of 123 carmine radish 'Hongxin 1' were collected for qRT-PCR analysis of miRNAs and their related 124 targets. All harvested tissues were immediately frozen in liquid nitrogen and stored at $-80^{\circ} \mathrm{C}$ for 125 qRT-PCR. Subsequently, using the mirVana ${ }^{\mathrm{TM}}$ miRNA Isolation Kit (Ambion) and Trizol 126 Reagent (Invitrogen, Nottingham, UK) kit according to the manufacturers' instructions, small 127 and total RNAs were isolated from each sample.

128 Gene annotation, enrichment analysis and cluster analysis of differentially 129 expressed transcription factors (DETFs)

Transcription factors (TFs) were obtained from the available 'Hongxin' carmine radish transcription profile data. Subsequently, the transcription factors (TFs) were subjected to GO annotation analysis using the Gene Ontology database (http://www.geneontology.org/) and to

133 KEGG pathway enrichment analysis using KOBAS software(Xie et al. 2011). Differentially expressed transcription factors (DETFs) were then screened by noiseqbio (Tarazona et al. 2012) and identified using a corrected P-value $<0.05$ between each set of compared samples (fold changes in the expression levels of genes in six radish cultivars, including 'IE_root', 'FE_root', 'BS_root', 'IFS_root', 'FBS_root' and 'PS_root', were identified by comparison with 'SS_root'- 'IE_root' Vs 'SS_root', 'FE_root' Vs 'SS_root', 'BS_root' Vs 'SS_root', 'IFS_root' Vs 'SS_root', 'FBS_root' Vs 'SS_root' and 'PS_root' Vs 'SS_root'). Differentially expressed transcription factors (DETFs) were then clustered according to their expression patterns in the dynamic growth stages of carmine radish and plotted using the neighbor-joining cluster method through a homemade R script.

\section{Expression pattern analysis and regulatory pathway identification}

144

To better understand the regulatory networks involving the DETFs, we searched putative DETFs targeted by miRNAs collected from the literature published by Xixiang Li et al. (Sun et al. 2016) using psRNATarget (http://plantgrn.noble.org/psRNATarget/?function.3), and the parameters were set as follows: a maximum expectation of 3.5 and a target accessibility (UPE) of 50. Negative correlations between DETs and miRNAs were validated by quantitative real-time 149 PCR.

\section{Validation of DETFs related to anthocyanin biosynthesis using qRT-PCR}

151

152

153

154

155

156

157

158

To monitor all DETFs related to anthocyanin biosynthesis, eight differentially expressed transcription factors (DETFs, cluster_24061_AP2/ERF-ERF, cluster_24166_AP2/ERF-ERF, cluster_59539_GRAS, cluster_44972_B3, cluster_22927_NAC, cluster_10013_C2C2_Dof, cluster_5503_bHLH, cluster_12215_bHLH, cluster_8116_WRKY and cluster_16741_C3H) were selected for qRT-PCR (ABI 7500 real-time PCR System, United States) based on their expression patterns (Table S1). In addition, eight differentially expressed transcription factors (DETFs) related to miRNAs (cluster_10013_C2C2_Dof, cluster_23883_C2C2_Dof, cluster_17168_bHLH, cluster_16851_AP2/ERF-ERF, cluster_11321_AP2/ERF-ERF, 
159 cluster_19966_AP2/ERF-ERF, Cluster_6812_AP2/ERF-ERF, cluster_16203_AP2/ERF-ERF, 160 and cluster_16851_AP2/ERF-ERF) were also selected for validation using qRT-PCR. The 161 primers for the qRT-PCR experiments were designed using Primer 5.0 software, and the radish 162 actin gene was used as the reference gene (Table S2). First-strand cDNA synthesis was 163 performed with $1 \mu \mathrm{g}$ of total RNA from 'Hongxin 1' carmine radish using an M-MLV reverse 164 transcriptase (Promega). The amplification programs were run according to the standard protocol 165 of the ABI7500 system in triplicate as described by Jian et al. (2015) (Jian et al. 2015). The 166 relative quantitative method $\left(2^{-\triangle \Delta C T}\right)$ was used to calculate the fold changes in the expression 167 levels of target genes (Schefe et al. 2006).

\section{Validation of the Candidate miRNAs by qRT-PCR}

169

To validate the putative miRNAs related to anthocyanin biosynthesis, eight miRNAs, including miR408a, miR172d, miR1432-5p, miR1425-5p, miR166g, miR827a, miR172b and

171 miR165a-5p, were selected based on their corresponding DETFs. The primers for qRT-PCR experiments were designed using Primer 5.0 software, and the radish gene actin was used as a standard control (Table S2). First, using the One Step miRNA 1st cDNA Synthesis Kit (Shenggong, Chengdu, China), microRNA reverse transcription reactions were performed in an Eppendorf Mastercycler (Eppendorf North America, Westbury, NY) for 60 min at $37^{\circ} \mathrm{C}$ and for 5 min at $95{ }^{\circ} \mathrm{C}$; the samples were then stored at $4{ }^{\circ} \mathrm{C}$ until further use. The RT-PCRs were performed in a $10 \mu \mathrm{L}$ volume containing $1 \mu \mathrm{L}$ of the diluted reverse transcription product, $1 \mu \mathrm{L}$ of PCR buffer, $0.2 \mathrm{mM}$ dNTPs, $2.0 \mathrm{U}$ of EasyTaq DNA polymerase (TransGen Biotech, Beijing, China), $0.5 \mu \mathrm{M}$ specific miRNA primer and $0.5 \mu \mathrm{M}$ universal primer (5TTACCTAGCGTATCGTTGAC-3) on an Eppendorf Mastercycler. The amplification programs were performed according to the standard protocol of the ABI7500 system in triplicate as

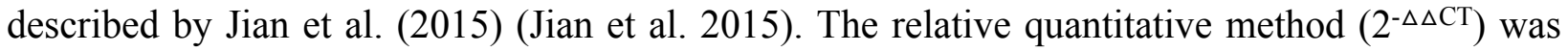
used to calculate the fold changes in the expression levels of target genes (Schefe et al. 2006).

\section{Data deposition}

All raw read sequences were deposited into the NCBI sequence read archive under the accession number PRJNA565866.

\section{Results}

Transcription factors (TFs) related to anthocyanin biosynthesis in the development of fleshy roots of carmine radish: A summary

Based on GO and KEGG pathway analyses, transcription factors (TFs) related to anthocyanin biosynthesis were identified in 'Hongxin 1' carmine radish. A total of 1,747 TFs in $64 \mathrm{TF}$ families related to anthocyanin biosynthesis in the dynamic developmental stages of fleshy roots were obtained from RNA-Seq analysis based on their DNA-binding domains. Of these TF 195 families, the AP2/ERF-ERF, WRKY, NAC, bHLH, bZIP, MYB-related, C3H, C2H2, GARP-G2like, MYB and GRAS families were found to be the most represented, with 125, 114, 84, 80, 78, $73,63,55,48,48$ and 43 members, respectively, and were expressed in at least one of the seven libraries (Fig. 1A). 
199

200

201

202

203

204

205

206

207

208

209

210

211

212

213

214

215

216

217

218

219

220

221

222

223

224

225

226

227

228

229

230

231

232

233

234

235

236

237

238

The results showed that $1,682,1,677,1,568,1,632,1,633,1,656$ and 1,586 TFs related to anthocyanin biosynthesis were expressed in fleshy roots from the seedling stage (SS), the initial expansion (IE) stage, the full expansion (FE) stage, the bolting stage (BS), the initial flowering stage (IFS), the full-bloom stage (FBS) and the podding stage (PS), respectively (Table S3). Of those, 1,415 (80.99\%) TFs were expressed in each of the seven libraries. Of those, 5 candidate TFs belonging to $4 \mathrm{TF}$ families (AP2/ERF-ERF, GRAS, NAC and B3) were found to exhibit opposite dynamic anthocyanin profiles in the fleshy roots of carmine radish, but 5 candidate TFs belonging to $4 \mathrm{TF}$ families (C2C2-Dof, bHLH, WRKY and C3H) were consistently found. Subsequently, those 10 putative TFs were selected and validated by qRT-PCR analysis. As shown in Fig. 1B, the results of qRT-PCR analysis were highly consistent with the expression profiles determined by RNASeq.

\section{Differentially expressed TFs (DETFs) related to anthocyanin biosynthesis} involved in the development of fleshy roots in carmine radish

A total of 161 DETFs were identified to be related to anthocyanin biosynthesis during the development of fleshy roots in carmine radish when compared with the SS_root group ( $\mathrm{p}$ value $<$ 0.05 , fold change $<-1$ or $>1$ ). In total, 116, 130, 136, 144, 141 and 136 DETFs were identified for 'IE_root_Vs_SS_root', 'FE_root' Vs 'SS_root', 'BS_root' Vs 'SS_root', 'IFS_root' Vs 'SS_root', 'FBS_root' Vs 'SS_root' and 'PS_root' Vs 'SS_root', respectively (Fig. 2A). In addition, these 161 DETFs were categorized into 43 families based on their DNA-binding domains (Table S4). Of those, the $A P 2 / E R F-E R F$ (31 members), WRKY (9 members), bHLH (11 members), $N A C$ (7 members), $M Y B$ (7 members) and HB-HD-zip (5 members) families were found to be the most represented in the development of fleshy roots in carmine radish. Among the DETFs, approximately 71 were commonly differentially expressed in any one stage compared with the SS_root group using a venny graph (Fig. 2B). Subsequently, 161 DETFs were categorized into 4 distinct clusters using the K-means clustering algorithm (Fig. 2C). Among them, class I contained 24 substantially altered DETFs that were identified in the almostwhole stage and included the AP2/ERF-ERF (4 numbers), AUX/IAA (1 number), bHLH (1 number), HD-ZIP (3 ), NAC (2 numbers) and WRKY (4 numbers) transcripts. A total of 45 genes belonging to class II showed slight dynamic changes during the development of fleshy roots. The expression patterns of $67 \mathrm{TFs}$ in class IV were found to oppose those in class I, as they were substantially changed in the almost-whole stage and included AP2/ERF-ERF (22 numbers), $b H L H$ (6 numbers), $M Y B$ ( 8 numbers) and GRAS (2 numbers) transcripts. Class III contained 25 genes that did not show significant trends throughout the development of fleshy roots. We further performed KOBAS analysis to annotate the DETFs in classes I and IV that are involved in the development of fleshy roots (Fig. 2C).

\section{Validation of DETFs targeted by miRNAs related to anthocyanin biosynthesis in} the development of fleshy roots in carmine radish

To investigate the functions of DETFs in carmine radish, the web-based program psRNATarget was selected to predict their related miRNAs. A total of 26 DETF transcripts, including $M Y B$, $W R K Y, b H L H, E R F, G R A S, N F-Y A, C 2 H 2-D o f$, and HD-ZIP, were predicted to be targets of 74 
239

240

241

242

243

244

245

246

247

248

249

250

251

252

253

254

255

256

257

258

259

260

261

262

263

264

265

266

267

268

269

270

271

272

273

274

275

276

277

278

miRNAs belonging to 25 miRNA families (Table S5). In addition, we found that some miRNAs were associated with more than one DETF transcript. For example, miR395b could target ERF (Cluster_7379) and WRKY (Cluster_8116). In addition, some transcripts were regulated by more than one miRNA. For instance, ERF (Cluster_7379) was targeted by miR395a, miR395b and miR399b-5p, and WRKY (Cluster_8116) was targeted by miR319-5p, miR395b and miR535b (Table S5). Of those, 8 DETF transcripts belonging to the C2C2-Dof, bHLH and ERF families and their 8 corresponding miRNAs were selected for qRT-PCR to verify their functions in the development of fleshy roots in carmine radish (Fig. 3A). The expression levels of miR408a, miR1432-5p and miR166g were down-regulated in the development of fleshy roots in carmine radish 'Hongxin 1', whereas those of their corresponding target genes C2C2-Dof, bHLH and $E R F$, respectively, were upregulated in the 'Hongxin 1' cultivar (Fig. 3B, 3D, 3G). The expression levels of miR172b, miR165-5p, miR172d, miR827a and miR1425-5p were increased, while those of their corresponding target genes C2C2-Dof and ERF, respectively, were decreased in the 'Hongxin 1' cultivar (Fig. 3C, E, F, H, I). The results showed that inversely correlated expression patterns were found between the miRNAs and their corresponding targets.

\section{Putative miRNA-target model associated with anthocyanin biosynthesis in carmine radish}

Some transcription factors were shown to function as important regulators of anthocyanin biosynthesis, including $M Y B, b H L H, W R K Y, E R F$ and $H D$-Zip. In addition, sucrose synthase, sugar/inositol transporter, and the WMBW complex were also found to potentially participate in anthocyanin biosynthesis. Here, based on the annotations of DETFs, we identified 26 transcripts of DETFs targeted by 74 miRNAs belonging to 25 miRNA families that might participate in anthocyanin biosynthesis in carmine radish (Table S5). Here, we propose a putative miRNAtarget model associated with anthocyanin biosynthesis in carmine radish (Fig. 4). The miR395, miR319, miR5720, miR1432 and miR408 families potentially targeted $W R K Y, M Y B$ and $b-H L H$ through the WMBW complex; the miR171, miR408 and miR172, miR167 families possibly targeted GRAS, C2C2-Dof and HD-ZIP to play roles in auxin-mediated signaling; and miR827, miR165/166, miR172 and miR395/miR396 targeted ERF to activate sucrose synthase. All three biological processes (involving the WMBW complex, auxin-mediated signaling and sucrose synthase) influence the expression of structural genes ( $P A L, C 4 H, 4 C L, C H S, F 3 H, D F R, A N S$ and $U F G T$ ) and subsequently modulate anthocyanin biosynthesis by forming a complex regulatory network.

\section{Discussion}

In plants, the MBW protein complex formed by the $R 2 R 3-M Y B, b H L H$, and $W D 40$ proteins could transcriptionally activate the promoters of structural genes related to anthocyanin synthesis (Xu et al. 2015). The complex is boosted by the participation of a WRKY transcription factor, which also confers specificity to other sets of target genes involved in, for example, vacuolar hyperacidification (Verweij et al. 2016). Previous studies showed that the expression of the MBW complex could be repressed by high levels of auxin to regulate the biosynthesis of

PeerJ reviewing PDF | (2019:07:39867:2:1:NEW 11 Oct 2019) 
279

280

281

282

283

284

285

286

287

288

289

290

291

292

293

294

295

296

297

298

299

300

301

302

303

304

305

306

307

308

309

310

311

312

313

314

315

316

317

318

anthocyanin(Ji et al. 2015; Liu et al. 2014). The $A R F$ and HD-ZIP transcription factor genes in the $A u x / I A A-A R F$ pathway were identified to regulate anthocyanin biosynthesis through auxinmediated signaling in apples(Wang et al. 2013). The HD-ZIP TF plays vital roles in regulating organ polarity in plants (Williams 2005). In addition, transcription factors (TFs) of the GRAS family have important control functions during primary and lateral root development, linking auxin signaling with cell specification and patterning, and are also involved in feedback regulation of local auxin homeostasis(Benjamins \& Scheres 2008). C2H2-Dof TFs are overrepresented in at least two auxin-inducible conditions in Arabidopsis(Peng et al. 2009). In this study, we demonstrated that HD-ZIPs and GRAS were targeted by miR167 and miR171, respectively, and that $C 2 C 2-D o f$ was targeted by miR408 and miR172; however, we did not identify a significant change in ARF in carmine radish. These results suggested that miR171, miR167, miR408 and miR172 might be involved in anthocyanin biosynthesis through auxinmediated signaling.

The MYB TFs have been identified as important regulators of anthocyanin biosynthesis through the MBW complex(Lai et al. 2014; Shen et al. 2014). Anthocyanin production could be effectively induced by AtPAP1 (AtMYB75) as well as by its orthologs in various plant species(Rowan et al. 2010; Zuluaga et al. 2008). Recently, $M Y B$ was identified as a target of many miRNAs in anthocyanin production in plants. An example is miR828 (Guan et al. 2014; Xie et al. 2013), which could cleave MYBL2 to improve anthocyanin accumulation in Arabidopsis(Yulong et al. 2016) and regulate two SlMYB transcripts for anthocyanin accumulation in tomato(Jia et al. 2015a). In this study, miR858, miR396, miR162 and ib-miR156 were identified to target $M Y B$ genes. In addition, a previous study showed that the transcription factor $b H L H$ can activate the expression of $R 2 R 3-M Y B$ and thereby activate or inhibit anthocyanin biosynthesis by binding to the promoters of DFR and UFGT (Feller et al. 2011; Xie et al. 2012). In this study, miR1432, miR408 and miR169 were identified to target $b H L H$.

In recent years, studies have demonstrated that sucrose synthase potentially participates in anthocyanin biosynthesis. A previous study showed that sucrose synthase could convert NDPglucose and d-fructose into NDP and sucrose. Subsequently, the increased sucrose content caused significant anthocyanin accumulation (Yozo et al. 2006). Moreover, Kuhn et al. demonstrated that miR854 could mediate the transport of sucrose through targeting the sugar/inositol transporter (Kühn et al. 2010). In addition, more evidence indicates that the ethylene signaling pathway links sucrose signaling to anthocyanin accumulation (Kwon et al. 2011; Shuichi et al. 2003). Here, we found that 9 miRNA families targeting 9 ERFs participate in anthocyanin biosynthesis through sucrose synthase, including miR172, miR166, miR399, miR1425, miR827, miR396, miR854, miR395 and miR863. Of those, 5 ERF DETFs and their related miRNAs were validated by qRT-PCR (Fig. 3b B, C, E, F and G). Recently, Yao et al. reported that the ethylene response factor (ERF)/APETALA2 (AP2) TF PyERF3 interacted with $P y M Y B 114$ and its partner PybHLH3 to coregulate anthocyanin biosynthesis in pears(Yao et al. 2017). We propose that ERFs might interact with the MBW protein complex through MYB and bHLH to coregulate anthocyanin biosynthesis in carmine radish, and further investigation of the

PeerJ reviewing PDF | (2019:07:39867:2:1:NEW 11 Oct 2019) 
319 MYB interaction with ERF to coordinately regulate fruit anthocyanin biosynthesis in carmine 320 radish is needed.

321 In this study, we propose a putative miRNA-target model associated with anthocyanin 322 biosynthesis in carmine radish (Fig. 4). The miR395, miR319, miR5720, miR1432 and miR408 323 miRNA families potentially targeted $W R K Y, M Y B$ and $b-H L H$ through the WMBW complex; the $324 \mathrm{miR} 171$, miR408 and miR172, miR167 families possibly targeted GRAS, C2C2-Dof and HD-ZIP 325 to play roles in auxin-mediated signaling; and miR827, miR165/166, miR172 and $326 \mathrm{miR} 395 / \mathrm{miR} 396$ targeted ERF to activate sucrose synthase. All three biological processes 327 (involving the WMBW complex, auxin-mediated signaling and sucrose synthase) influence the 328 expression of structural genes ( $P A L, C 4 H, 4 C L, C H S, F 3 H, D F R, A N S$ and UFGT) and subsequently modulate anthocyanin biosynthesis by forming a complex regulatory network. More interestingly, more ERF DETFs than WRKY, MYB and $b H L H$ DETFs were identified in this study. We proposed that sucrose synthase might be an important biological agent that influences the expression of structural genes ( $P A L, C 4 H, 4 C L, C H S, F 3 H, D F R, A N S$ and $U F G T$ ), thereby modulating anthocyanin biosynthesis in carmine radish; however, the mechanism by which ERFs modulate anthocyanin biosynthesis through sucrose synthase needs further investigation.

\section{Conclusion}

Here, 1,747 TFs belong to 64 TFs families were identified in a highly natural red pigment contained local cultivar named 'Hongxin 1', according to their DNA-binding domains. Of those, 26 transcripts of DETFs targeted by 74 miRNAs belong to 25 miRNAs family were identified, such as $M Y B, W R K Y, b H L H, E R F, G R A S, N F-Y A, C 2 H 2-D o f, H D-Z I P$, etc. Finally, 8 DETFs transcripts belong to $C 2 C 2-D o f, b H L H$ and $E R F$ family and their 8 corresponding miRNAs were selected for qRT-PCR to verify their function related to anthocyanin biosynthesis. Our findings proposed that sucrose synthase might act as important regulators to modulate anthocyanin biosynthesis in carmine radish, through induce several miRNAs (miR165a-5p, miR172b, miR827a, miR166g and miR1432-5p) targeted different ERFs, compared with traditional WMBW complex biological process.

\section{Not applicable.}

\section{References}

Anju Bajpai, Kasim Khan, M. Muthukumar, S. Rajan, and Singh NK. 2017. Molecular analysis of anthocyanin biosynthesis pathway genes and their differential expression in mango peel. Genome 61:157.

Aza-González C, Herrera-Isidrón L, Núñez-Palenius HG, Vega OMDL, and Ochoa-Alejo N. 2012. Anthocyanin accumulation and expression analysis of biosynthesis-related genes during chili pepper fruit development. Biologia Plantarum 57:49-55.

Benjamins R, and Scheres B. 2008. Auxin: The Looping Star in Plant Development. Annual Review of Plant Biology 59:443-465.

Dao TTH, Linthorst HJM, and Verpoorte R. 2011. Chalcone synthase and its functions in plant resistance. 
Phytochemistry Reviews 10:397-412.

Feller A, Machemer K, Braun EL, and Grotewold E. 2011. Evolutionary and comparative analysis of MYB and bHLH plant transcription factors. Plant Journal for Cell \& Molecular Biology 66:94-116.

Gou JY, Felippes FF, Liu CJ, Weigel D, and Wang JW. 2011. Negative Regulation of Anthocyanin Biosynthesis in Arabidopsis by a miR156-Targeted SPL Transcription Factor. Plant Cell 23:1512-1522.

Guan X, Pang M, Nah G, Shi X, Ye W, Stelly DM, and Chen ZJ. 2014. miR828 and miR858 regulate homoeologous MYB2 gene functions in Arabidopsis trichome and cotton fibre development. Nature Communications 5:3050.

He J, and Giusti MM. 2010. Anthocyanins: Natural Colorants with Health-Promoting Properties. Annual Review of Food Science \& Technology 1:163.

Ji X-H, Wang Y-T, Zhang R, Wu S-J, An M-M, Li M, Wang C-Z, Chen X-L, Zhang Y-M, and Chen X-S. 2015. Effect of auxin, cytokinin and nitrogen on anthocyanin biosynthesis in callus cultures of red-fleshed apple (Malus sieversiif.niedzwetzkyana). Plant Cell Tissue \& Organ Culture 120:325-337.

Jia X, Shen J, Liu H, Li F, Ding N, Gao C, Pattanaik S, Patra B, Li R, and Yuan L. 2015a. Small tandem target mimic-mediated blockage of microRNA858 induces anthocyanin accumulation in tomato. Planta 242:283293.

Jia XY, Liu H, Shen J, Fang LI, Ding N, Sun Y, Gao CY, and Run-Zhi LI. 2015b. Negative Regulation of Anthocyanin Biosynthesis in Tomato by MicroRNA828 Under Phosphate Deficiency. Scientia Agricultura Sinica. 48(15):2911-2924.

Jian G, Mao L, Zhang C, Hua P, Lin H, Shen Y, Zhao M, Pan G, and Zhang Z. 2015. A putative pathogen-resistant regulatory pathway between MicroRNAs and candidate target genes in maize. Journal of Plant Biology 58:211-219.

Khoo HE, Azlan A, Tang ST, and Lim SM. 2017. Anthocyanidins and anthocyanins: colored pigments as food, pharmaceutical ingredients, and the potential health benefits. Food \& Nutrition Research 61:1361779.

Lai B, Li XJ, Hu B, Qin YH, Huang XM, Wang HC, and Hu GB. 2014. LcMYB1 Is a Key Determinant of Differential Anthocyanin Accumulation among Genotypes, Tissues, Developmental Phases and ABA and Light Stimuli in Litchi chinensis. Plos One 9:e86293.

Latchman DS. 1993. Transcription factors: an overview. International Journal of Biochemistry \& Cell Biology 74:417-422.

Liu Z, Shi M-Z, and Xie D-Y. 2014. Regulation of anthocyanin biosynthesis inArabidopsis thalianaredpap1-Dcells metabolically programmed by auxins. Planta 239:765-781.

Peng Z, Zhou X, Li L, Yu X, Li H, Jiang Z, Cao G, Bai M, Wang X, and Jiang C. 2009. Arabidopsis Hormone Database: a comprehensive genetic and phenotypic information database for plant hormone research in Arabidopsis. Nucleic Acids Research 37:D975-D982.

Qi T, Song S, Ren Q, Wu D, Huang H, Chen Y, Fan M, Peng W, Ren C, and Xie D. 2011. The Jasmonate-ZIMDomain Proteins Interact with the WD-Repeat/bHLH/MYB Complexes to Regulate Jasmonate-Mediated Anthocyanin Accumulation and Trichome Initiation in Arabidopsis thaliana. Plant Cell 23:1795-1814.

Rowan DD, Cao M, Linwang K, Cooney JM, Jensen DJ, Austin PT, Hunt MB, Norling C, Hellens RP, and Schaffer RJ. 2010. Environmental regulation of leaf colour in red 35S:PAP1 Arabidopsis thaliana. New Phytologist 182:102-115.

Schefe JH, Lehmann KE, Buschmann IR, Unger T, and Funke-Kaiser H. 2006. Quantitative real-time RT-PCR data analysis: current concepts and the novel "gene expression'sCTdifference" formula. Journal of Molecular Medicine 84:901-910.

Shen X, Zhao K, Liu L, Zhang K, Yuan H, Liao X, Wang Q, Guo X, Li F, and Li T. 2014. A Role for PacMYBA in ABA-Regulated Anthocyanin Biosynthesis in Red-Colored Sweet Cherry cv. Hong Deng (Prunus avium L.). Plant \& Cell Physiology 55:862.

Tarazona S, García F, Ferrer A, Dopazo J, and Conesa A. 2012. NOIseq: a RNA-seq differential expression method robust for sequencing depth biases. University of Southampton 17(B):18.

Verweij W, Spelt CE, and Bliek M. 2016. Functionally Similar WRKY Proteins Regulate Vacuolar Acidification in 
409

410

411

412

413

414

415

416

417

418

419

420

421

422

423

424

425

426

427

428

429

430

431

432

433

434

435

436

437

438

439

440

441

442

443

444

445

446

447

448

449

450

451

Petunia and Hair Development in Arabidopsis. Plant Cell 28:786.

Wang S, Hagen G, and Guilfoyle TJ. 2013. ARF-Aux/IAA interactions through domain III/IV are not strictly required for auxin-responsive gene expression. Plant Signaling \& Behavior 8: e2452

WILLIAMS L. 2005. Regulation of Arabidopsis shoot apical meristem and lateral organ formation by microRNA miR166g and its AtHD-ZIP target genes. Development 132:3657-3668.

Xie C, Mao X, Huang J, Ding Y, Wu J, Dong S, Kong L, Gao G, Li C-Y, and Wei L. 2011. KOBAS 2.0: a web server for annotation and identification of enriched pathways and diseases. Nucleic Acids Research 39:316322.

Xie XB, Li S, Zhang RF, Zhao J, and Hao YJ. 2012. The bHLH transcription factor MdbHLH3 promotes anthocyanin accumulation and fruit coloration in response to low temperature in apples. Plant Cell \& Environment 35:1884-1897.

Xie Y, Sun Y, and Dan-Ning LI. 2013. MicroRNA828 Negatively Regulates Sucrose-Induced Anthocyanin Biosynthe-sis in Arabidopsis. Plant Physiology Journal 49:188-194.

Xu W, Dubos C, and Lepiniec Lc. 2015. Transcriptional control of flavonoid biosynthesis by MYB-bHLH-WDR complexes. Trends in Plant Science 20:176-185.

Yang F, Cai J, Yang Y, and Liu Z. 2013. Overexpression of microRNA828 reduces anthocyanin accumulation inArabidopsis. Plant Cell Tissue \& Organ Culture 15:159-167.

Yousuf B, Gul K, Wani AA, and Singh P. 2015. Health Benefits of Anthocyanins and Their Encapsulation for Potential Use in Food Systems: A Review. Crit Rev Food Sci Nutr 56:2223-2230.

Yulong, Wang, Yiqing, Wang, Zhaoqing, Song, Huiyong, and Zhang. 2016. Repress,on of MYBL2 by Both microRNA858a and HY5 Leads to the Activation of Anthocyanin Biosynthetic Pathway in Arabidopsis. Molecular Plant 9:1395-1405.

Yusuf D, Butland SL, Swanson MI, Bolotin E, Ticoll A, Cheung WA, Zhang XYC, Dickman CT, Fulton DL, and Lim JS. 2012. The Transcription Factor Encyclopedia. Genome Biology 13:R24.

Zhou H, Lin-Wang K, Wang H, Gu C, Dare AP, Espley RV, He H, Allan AC, and Han Y. 2015. Molecular genetics of blood-fleshed peach reveals activation of anthocyanin biosynthesis by NAC transcription factors. Plant Journal 82:105-121.

Zuluaga DL, Gonzali S, Loreti E, Pucciariello C, and Perata P. 2008. Arabidopsis thaliana MYB75/PAP1 transcription factor induces anthocyanin production in transgenic tomato plants. Functional Plant Biology 35:606-618.

\section{Supplemental materials}

Figure S1: Dynamics anthocyanidin profiles conducted in the development of fleshy roots of carmine radish.

Table S1: List of primers for qRT-PCR analysis of 10 candidate DETFs related to anthocyanin biosynthesis involved in the dynamics growing stages of fleshy roots in carmine radish.

Table S2: List of primers for qRT-PCR analysis of 8 candidate DETFs, as well as their related miRNAs association with anthocyanin biosynthesis involved in the dynamics growing stages of flesh roots in carmine radish.

Table S3: Genome-wide identification of Transcription factors (TFs) related to anthocyanin biosynthesis involved in the dynamics growing stages of flesh roots in carmine radish.

Table S4: Differential expressed Transcription factors (DETFs) related to anthocyanin biosynthesis identified involved in the dynamics growing stages of flesh roots in carmine radish.

PeerJ reviewing PDF | (2019:07:39867:2:1:NEW 11 Oct 2019) 
452 Table S5: Prediction of miRNAs related to differential expressed transcription factors (DETFs) 453 association with anthocyanin biosynthesis involved in the dynamics growing stages of flesh roots 454 in carmine radish. 


\section{Figure 1}

Figure 1 Identification and qRT-PCR validation of Transcription factors (TFs) related to anthocyanin biosynthesis in the development of fleshy roots in carmine radish

(A) Identification of transcription factors (TFs) related to anthocyanin biosynthesis involved in the development of fleshy roots in 'Hongxin 1' carmine radish (B) Validate of candidate TFs related to anthocyanin biosynthesis involved in the dynamics growing stages of fleshy roots in carmine radish using qRT-PCR and then correlation between RNA-seq and qPCR data were conducted. Each RNA-seq expression data was plotted against that from quantitative realtime PCR and fit into a linear regression. Both $\mathrm{x}$ - and $\mathrm{y}$-axes were shown in log2 scale and each color represented a different gene. 


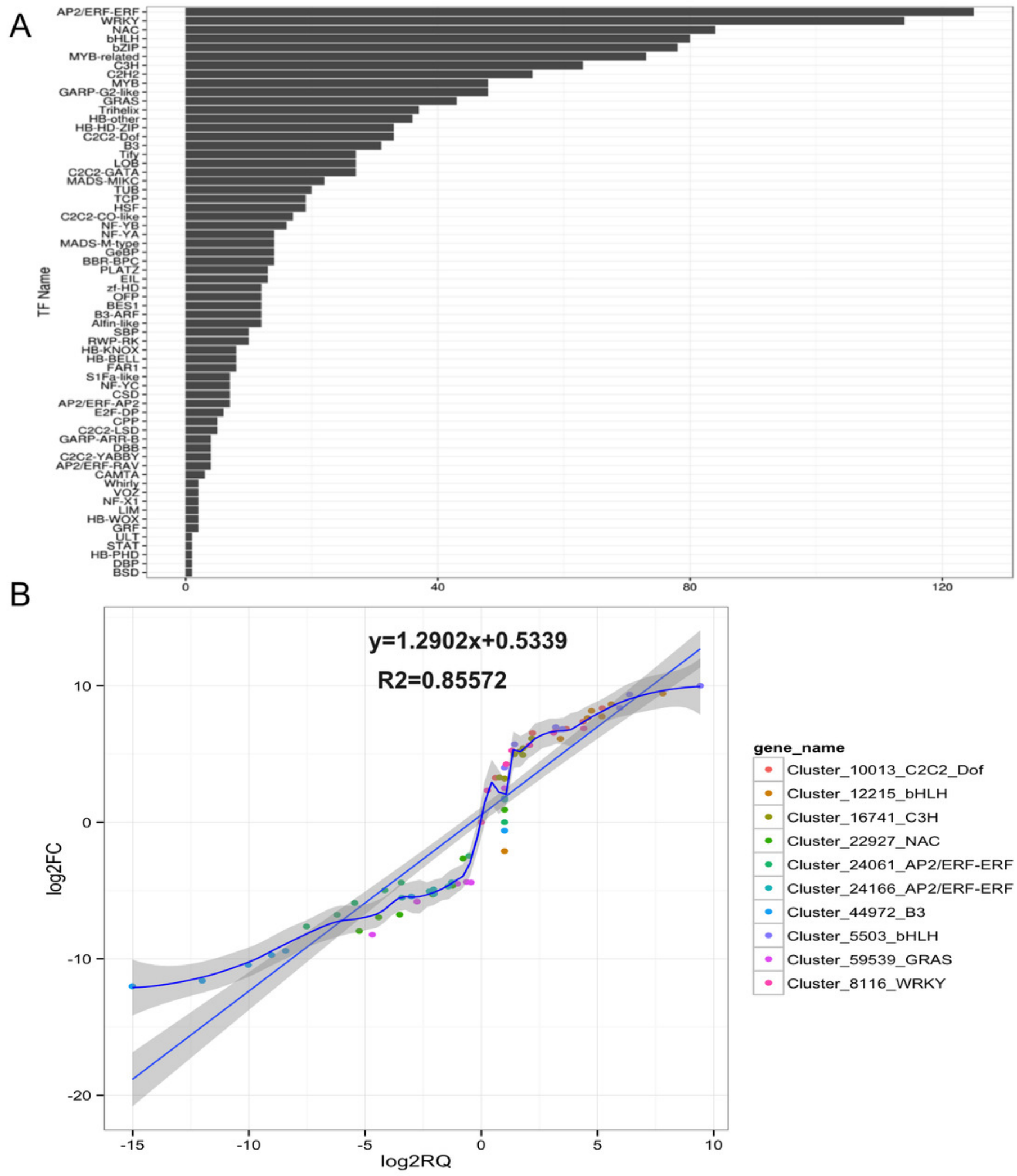




\section{Figure 2}

Figure 2 Identification of DETFs related to anthocyanin biosynthesis and their expression patterns in the development of fleshy roots of carmine radish.

(A) Statistic of differentially expression transcription factors (including up-regulated and down-regulated in each comparison groups) related to anthocyanin biosynthesis in the dynamics growing stages of fleshy roots ('IE_root', 'FE_root', 'BS_root', 'IFS_root', 'FBS_root' and 'PS_root'), compared with 'SS_root' group. (B) Venny graph of co-modulated DEFTs (Common DETFs related to anthocyanin biosynthesis in carmine radish). (C) Clustering and heat map of common DETFs related to anthocyanin biosynthesis based on the expression profiles in the dynamics growing stages of fleshy roots ('IE_root', 'FE_root', 'BS_root', 'IFS_root', 'FBS_root' and 'PS_root'), compared with 'SS_root' group. 


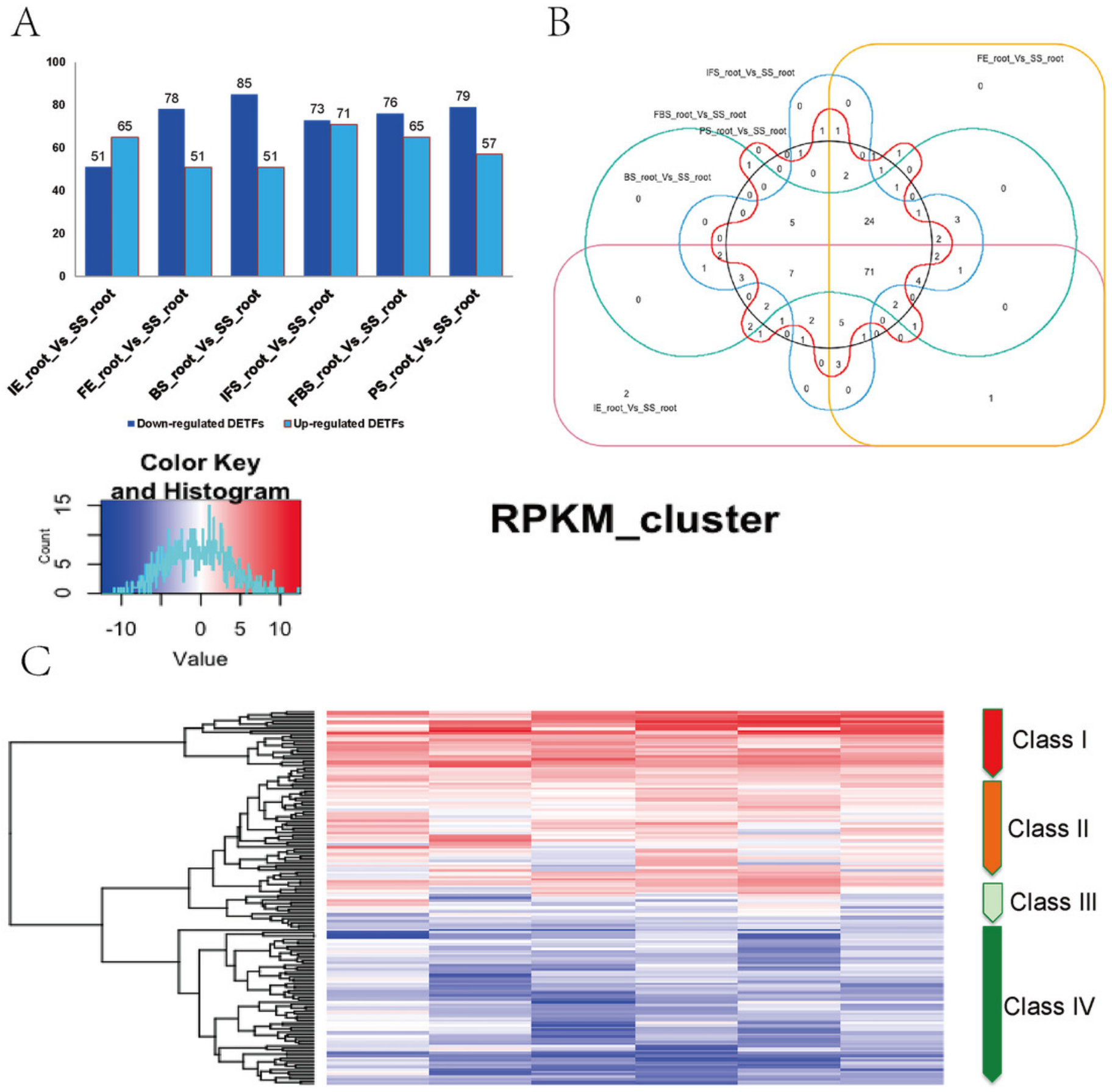




\section{Figure 3}

Figure 3 Predict of miRNAs regulated by DEFTs related to anthocyanin biosynthesis, as well as validate the DETFs and their related miRNAs in the development of fleshy roots in carmine radish.

(A) Predict of miRNAs regulated by DEFTs using psRNATarget. The miRNA target sites (red) with the nucleotide positions of DEFTs transcripts are shown. The RNA sequence of each complementary site from $5^{\prime}$ to $3^{\prime}$ and the predicted miRNA sequence from $3^{\prime}$ to $5^{\prime}$ are indicated in the expanded regions. (B) qRT-PCR analysis of miRNAs and DETFs expressed in different samples in the development of fleshy roots in carmine radish. Standard error bars are provided for three biological repeats. 
A

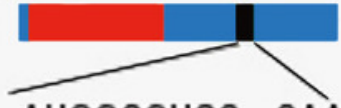

AUGGCGUCC.-.-CAAGAUUCCCAA

|||\|ा| ||\|\|\|\|\|\| 3'CGUCGUAGUAGUUCUAAGGGUU5' miR172d 659-677

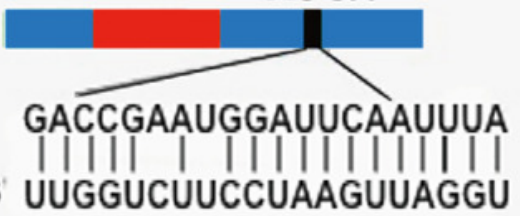

\section{Cluster16851 \\ (AP2/ERF-ERF)}

Cluster_23883

(C2C2-Dof)

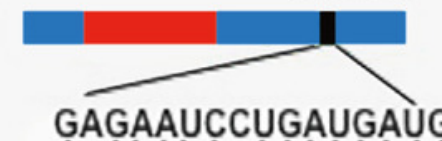

Cluster6812

(AP2/ERF-ERF)

AGAAUCCUGAUGAÜGCUGC

3. CACUUAGAAUUACUAUGACG 5 ' miR172b

$1325-1348$

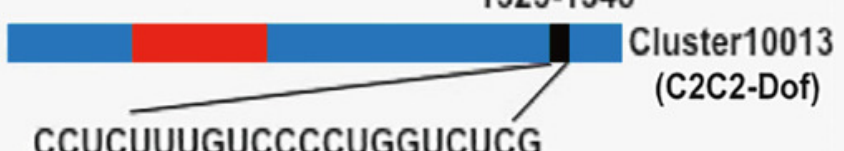

CCUCUUUGUCCCCUGGUCUCG

5' miR1425-5p 3' GGAGAGAACAAGGGACAGAGA 5' miR408a

3 UUGGUCUUCCUAAGUUAGGU

298-318

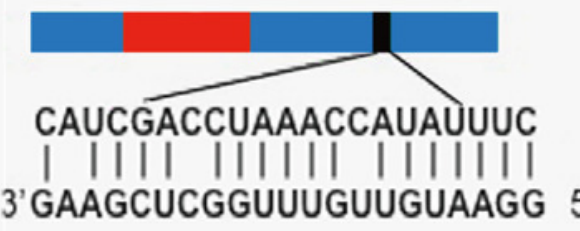
$1190-1210$

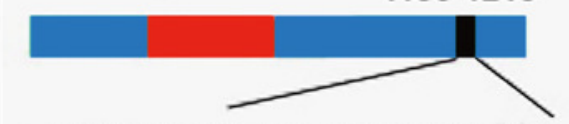

UAUUUGUUGAUGUUCAUGUAA

Cluster_11321

(AP2/ERF-ERF)

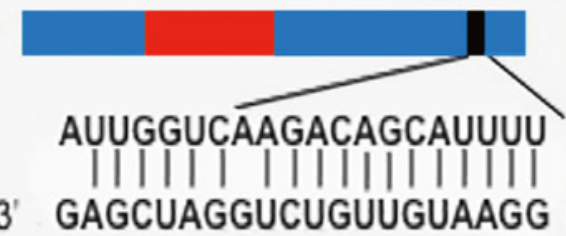

Custer16203

(AP2/ERF-ERF)

5' miR166g

Cluster_19966

(AP2/ERF-ERF)

3' AUAAACAACUACCAGUAGAUU

miR827a
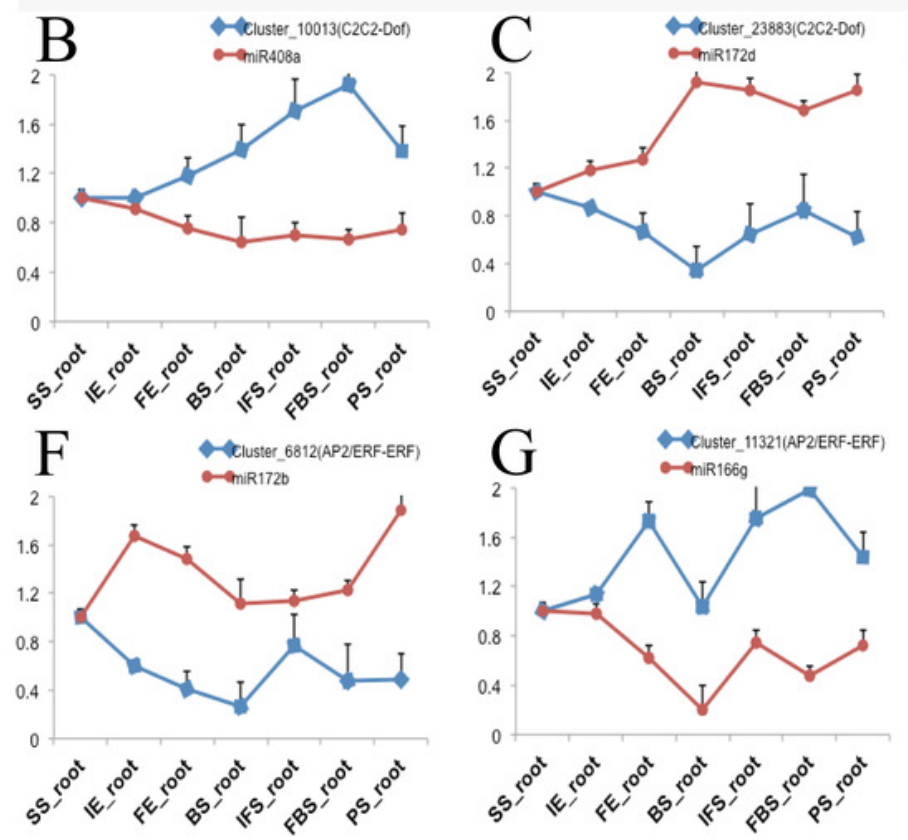

D

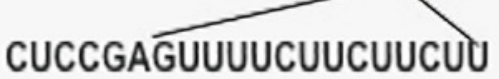

963.983
|| || || || | || || || ||

3' GAGGCUCAGUAGAAGAGGAU 5' miR1432-5p

\section{(bHLH)}
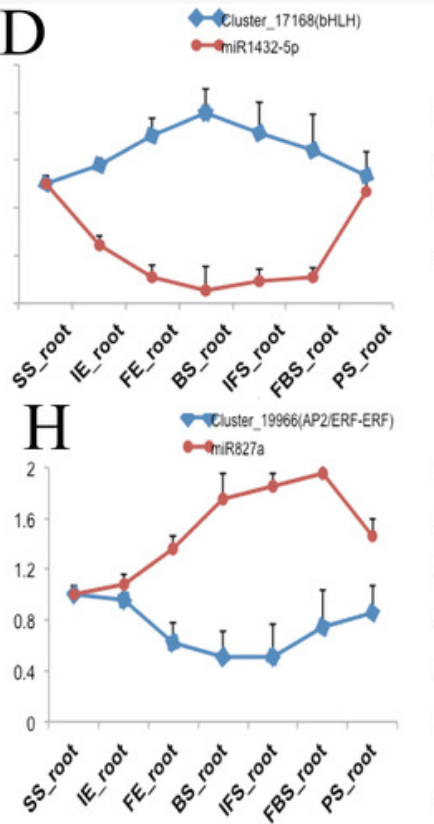

E - -elluster_16203(AP2/ERF-ERF)
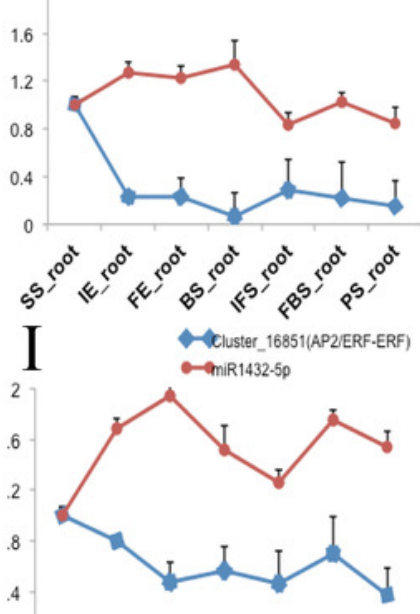

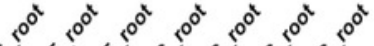

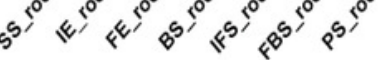




\section{Figure 4}

Figure 4 A proposed model of transcription factor related to anthocyanin biosynthesis targeted by miRNAs were involved in the development of fleshy roots in carmine radish.

The genes in blue box denote DETs identified in the present research, as well as their responding miRNAs denoted in green box.

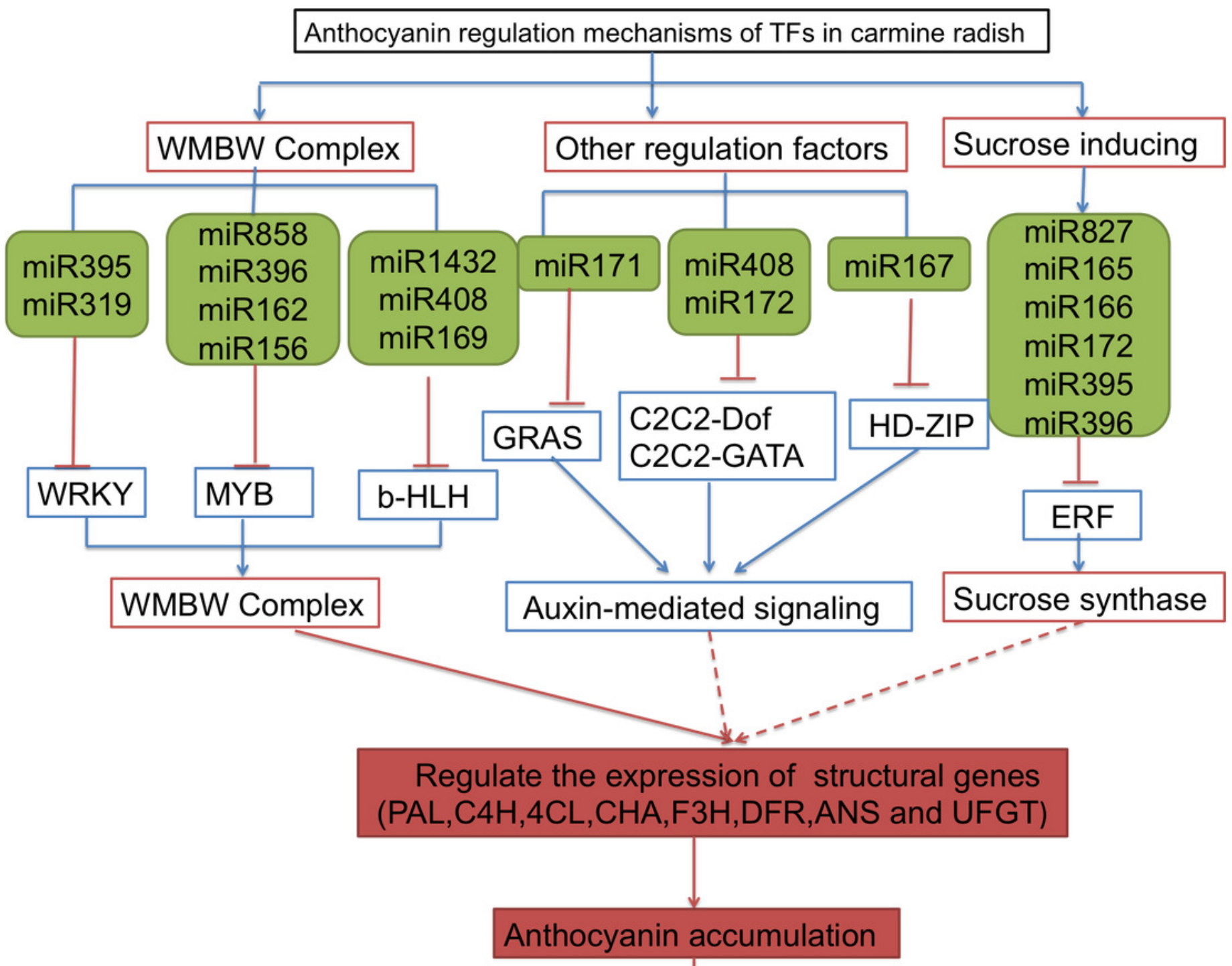

